PHYSICAL REVIEW E 71, 056613 (2005)

\title{
Polarization instability, steering, and switching of discrete vector solitons
}

\author{
Rodrigo A. Vicencio and Mario I. Molina \\ Departamento de Física, Facultad de Ciencias, Universidad de Chile, Casilla 653, Santiago, Chile \\ Yuri S. Kivshar \\ Nonlinear Physics Centre and Centre for Ultra-high bandwidth Devices for Optical Systems (CUDOS), Research School of Physical \\ Sciences \\ and Engineering, Australian National University, Canberra, ACT 0200, Australia
}

(Received 13 September 2004; published 24 May 2005)

\begin{abstract}
We study the dynamics of discrete vector solitons in arrays of weakly coupled birefringent optical waveguides with cubic nonlinear response. We start with a modulational instability analysis, followed by approximate analytical solutions in the form of strongly localized modes. Next, we compute the effective Peierls-Nabarro potential for these modes and obtain the spatial average of the power transfer between both polarizations modes as a function of their relative phase. Finally, we combine the concepts of polarization mode instability with discreteness-induced beam trapping by the array, and demonstrate numerically the amplification of a weak signal by a strong pump of the other polarization, combined with simultaneous discretized all-optical switching.
\end{abstract}

DOI: 10.1103/PhysRevE.71.056613

PACS number(s): 42.65.Tg, 42.82.Et, 42.65.Sf

\section{INTRODUCTION}

The study of discrete spatial solitons has attracted a lot of attention during the last years because of many successful experimental observations of such solitons in different lattice systems, including arrays of weakly coupled optical waveguides in AlGaAs semiconductor structures [1], optically induced photonic lattices in photorefractive materials [2], and voltage-controlled waveguide arrays in nematic liquid crystals [3] (see also a number of comprehensive review papers [4]).

Discrete spatial solitons have been first introduced as spatially localized nonlinear modes of weakly coupled optical waveguides, which can exist due to a balance between discrete diffraction in the array and nonlinear response of the waveguide material [5]. A standard theoretical approach to study discrete spatial optical solitons is based on the derivation of an effective discrete nonlinear model and the analysis of its stationary localized solutions-discrete localized modes [6].

As localized modes of discrete nonlinear models, such discrete solitons appear in many diverse areas of physics [7]. For example, they have been theoretically predicted to exist in many systems, including the Bose-Einstein condensates loaded onto an optical lattice [8], electronic transport in crystals, biopolymer chains [9], etc. More importantly, the discrete solitons (also called "discrete breathers") have been observed in a number of different physical systems such as low-dimensional molecular crystals [10], antiferromagnetic chains [11], arrays of Josephson junctions [12], and micromechanical oscillator arrays [13].

In optical waveguide arrays, both propagation and steering of scalar discrete solitons have been studied more extensively because of potential applications for all-optical switching devices. These studies include, for example, trapping, reflection, and refraction of discrete solitons in waveguide arrays with defects [14], and soliton switching in waveguide arrays with quadratic nonlinear response [15]. Recently, we suggested $[16,17]$ to control multiport switching of discrete solitons in waveguide arrays by engineering the coupling between the neighboring waveguides: this induces a change of the dynamic properties of the array through the modification of the effective Peierls-Nabarro (PN) potential, a nonlinear discreteness-induced potential that is responsible for the transverse dynamics and steering of discrete solitons in waveguide arrays (see, e.g., Refs. $[18,19])$. This year Kartashov et al. [20] showed that in the quasicontinuous limit a similar digital switching effect can be observed opening many possibilities for observation in real experiments.

Most of the earlier studies analyzed the properties of $s c a-$ lar discrete solitons. However, the nonlinear cubic waveguides that constitute the array are always birefringent; thus we can expect a very rich dynamics via coupling between the orthogonal polarizations (see, e.g., Ref. [6]). One of the major results in the study of the polarization dynamics in homogeneous systems is the existence of polarization instability demonstrated for birefringent optical fibers [21,22] and planar optical waveguides [23-25]. As a consequence of this instability, the fast modes become unstable whereas slow modes remain stable, and the instability dynamics results in the energy exchange between the polarizations. The definitions of the transverse electric (TE) modes and the transverse magnetic (TM) modes as slow or fast depends on the system geometry. In AlGaAs optical waveguides the TE mode is slow and, therefore, it constitutes a stable mode [25].

Vector solitons have been suggested for different schemes of all-optical switching, e.g., by employing collisions between orthogonally polarized solitons with [26] or without [27] four-wave mixing (FWM) effects. The FWM effect is responsible for the energy exchange between the two orthogonal (TE and TM) polarization modes. The theory of discrete vector solitons developed so far does not include the analysis of the FWM effects (as an example, see Ref. [28]). 


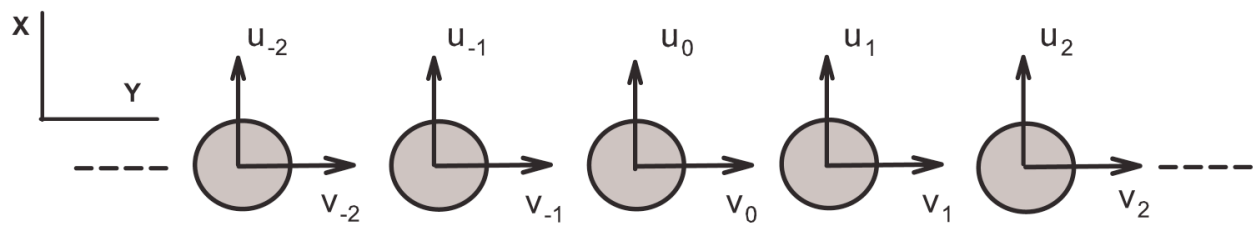

FIG. 1. Schematic head-on view of the waveguide array.

However, the first experimental studies of the vectorial interactions in waveguide arrays and observation of discrete vector solitons [29] suggest the importance of the FWM effects by demonstrating that the initial phase between the TE and TM modes defines the energy exchange between the modes due to coupling between two orthogonal polarizations.

In this paper, we study the dynamics of discrete vector solitons in arrays of weakly coupled birefringent optical waveguides with cubic nonlinear response, taking into consideration recent experimental observations [29]. First, we find approximate analytical solutions for strongly localized vectorial modes. Using these modes, we study the energy transfer between the orthogonal polarizations and polarization mode instability, and also calculate the effective PN potential. Finally, we employ the concept of the polarization instability and discreteness-induced beam trapping by the array and demonstrate a novel approach to all-optical soliton switching, amplification of a weak signal by a strong pump of the other polarization combined with simultaneous discretized switching.

\section{VECTOR DISCRETE NONLINEAR SCHRÖDINGER EQUATIONS}

We describe propagation of light in arrays of weakly coupled birefringent waveguides [29] by using the couplemode theory combined with the slowly varying envelope approximation. Then, the mode dynamics is described by discrete dynamical equations of the form,

$$
i \frac{d u_{n}}{d z}+u_{n}+C\left(u_{n+1}+u_{n-1}\right)+\left|u_{n}\right|^{2} u_{n}+A\left|v_{n}\right|^{2} u_{n}+B v_{n}^{2} u_{n}^{*}=0
$$

$i \frac{d v_{n}}{d z}-v_{n}+C\left(v_{n+1}+v_{n-1}\right)+\left|v_{n}\right|^{2} v_{n}+A\left|u_{n}\right|^{2} v_{n}+B u_{n}^{2} v_{n}^{*}=0$

where $u_{n}$ and $v_{n}$ are the normalized envelopes of the TE and TM electric field components, respectively, $z$ is the propagation distance, $C$ is the coupling parameter being the same for both polarizations (both modes have similar transversal extensions), the coefficients $A$ and $B$ characterize the crossphase modulation and FWM effects, respectively (being both normalized to the self-focusing term). Figure 1 shows a schematic transversal view of the waveguide array, where the orthogonal polarizations have been chosen along the $x$ and $y$ directions. For the $\mathrm{Al}_{x} \mathrm{Ga}_{1-x} \mathrm{As}$ waveguide arrays [29] the parameters are $A=1, B=0.5$, and the TE mode corresponds to the slow wave whereas the TM mode-to the fast wave. To consider the nonlinear Kerr effect in a experiment using $\mathrm{Al}_{x} \mathrm{Ga}_{1-x} \mathrm{As}$, it is necessary to take the photon energies below one-half of the semiconducting band gap energy $(\hbar \omega$
$\left.<E_{g} / 2\right)$. In such material this energy corresponds to a $\lambda$ $\approx 1.5 \mu \mathrm{m}$, where the linear and nonlinear absorption effects are minimized [30].

The normalized power and Hamiltonian are given by

$$
P=P_{u}+P_{v}=\sum_{n}\left(\left|u_{n}\right|^{2}+\left|v_{n}\right|^{2}\right),
$$

$$
\begin{aligned}
H= & -\sum_{n}\left(C\left(u_{n}^{*} u_{n+1}+u_{n} u_{n+1}^{*}+v_{n}^{*} v_{n+1}+v_{n} v_{n+1}^{*}\right)+\left|u_{n}\right|^{2}-\left|v_{n}\right|^{2}\right. \\
& \left.+\frac{1}{2}\left(\left|u_{n}\right|^{4}+\left|v_{n}\right|^{4}\right)+A\left|u_{n}\right|^{2}\left|v_{n}\right|^{2}+\frac{B}{2}\left(v_{n}^{2} u_{n}^{* 2}+u_{n}^{2} v_{n}^{* 2}\right)\right),
\end{aligned}
$$

and they are conserved by the dynamics. These quantities play an important role in the theoretical analysis, in checking numerical accuracy, and in the real power estimation for the switching-amplification process (for $\mathrm{Al}_{x} \mathrm{Ga}_{1-x} \mathrm{As}$ waveguides $\left.[29], P_{\text {real }} \approx 56 P[W]\right)$.

Equations (1)-(3) indicate explicitly that the slow and fast modes are not equivalent. This is confirmed, for example, by numerical simulations of the nonlinear dynamics of both slow and fast modes. Moreover, it is easy to see that the Hamiltonian for the slow wave is lower than for the fast wave. This suggests that the fast wave may become unstable with respect to its transformation into the slow wave. In the next section, we study this issue in detail.

\section{MODULATIONAL INSTABILITY}

We study modulational instability (MI) of finite-amplitude solutions of the discrete vectorial model. We employ a standard analytical approach [31] and consider the evolution of a weakly perturbed finite-amplitude plane waves $u_{n}(z)=\left[u_{0}\right.$ $\left.+\delta u_{n}(z)\right] e^{i\left(q_{u} n+k_{u} z\right)}, v_{n}(z)=\left[v_{0}+\delta v_{n}(z)\right] e^{i\left(q_{v} n+k_{v} z\right)}$, where $u_{0}$ and $v_{0}$ are the mode amplitudes, $\delta u_{n}$ and $\delta v_{n}$ are small perturbation functions, $q_{u}$ and $q_{v}$ are the transverse components of the wave vectors, and $k_{u}$ and $k_{v}$ are the propagation constants of the TE and TM modes, respectively. The perturbation functions are taken of the form $\delta u_{n}(z)=u_{1} e^{i(Q n-K z)}$ $+u_{2}^{*} e^{-i\left(Q n-K^{*} z\right)}, \delta v_{n}(z)=v_{1} e^{i(Q n-K z)}+v_{2}^{*} e^{-i\left(Q n-K^{*} z\right)}$, where the amplitudes $u_{1}, u_{2}^{*}, v_{1}$, and $v_{2}^{*}$, are assumed to be small, where $Q$ and $K$ are the wave vector parameters of the perturbation. The modulational instability gain $G$ can be defined as $G$ $\equiv \operatorname{Im}[K]$. The instability regions corresponds to the condition $G>0$, while for $G<0$, the plane wave solutions are stable. The results of the MI analysis suggest that when plane waves are unstable, we can expect the formation of spatially localized structures in the waveguide array, the so-called discrete vector solitons.

After substituting these weakly perturbed plane waves into the dynamical Eqs. (1) and after applying a standard 


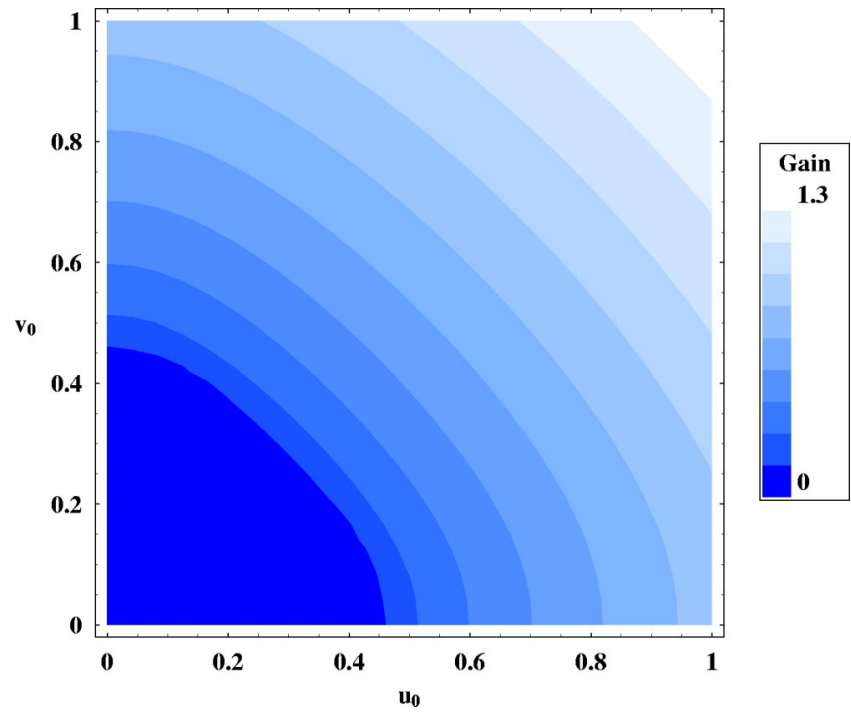

FIG. 2. (Color online) Gain of modulational instability as a function of the mode amplitudes, $u_{0}$ and $v_{0}$, for $C=0.1$. No instability exists in the darkest area.

analysis [31], we obtain a simple quadratic equation for $K^{2}$, $K^{4}+a_{1} K^{2}+a_{2}=0$, where

$$
\begin{aligned}
a_{1} \equiv & -\left(f^{2}+g^{2}\right)+\left(1+B^{2}\right) u_{0}^{4}-4 B(2 A+2 B-1) u_{0}^{2} v_{0}^{2} \\
& +\left(1+B^{2}\right) v_{0}^{4}
\end{aligned}
$$

and

$$
\begin{aligned}
a_{2} \equiv & \left\{\left(f-u_{0}^{2}\right)\left(g-B u_{0}^{2}\right)-\left[f+B g+\left(3 B^{2}-1\right) u_{0}^{2}\right] v_{0}^{2}+B v_{0}^{4}\right\} \\
& \times\left\{\left(f+u_{0}^{2}\right)\left(g+B u_{0}^{2}\right)+\{f+B g-[(2 A+B)(2 A+3 B)\right. \\
& \left.\left.-1] u_{0}^{2}\right\} v_{0}^{2}+B v_{0}^{4}\right\},
\end{aligned}
$$

where $f=-4 C \sigma_{u} \sin ^{2}(Q / 2)+u_{0}^{2}-B v_{0}^{2}, \quad g=-4 C \sigma_{v} \sin ^{2}(Q / 2)$ $+v_{0}^{2}-B u_{0}^{2}$, where $\sigma_{u}=\cos q_{u}$ and $\sigma_{v}=\cos q_{v}$ describe the structure of the carrier waves in both the components. In particular we are interested in unstaggered modes, that imply to take $q_{u}=q_{v}=0$ (for staggered modes we need to take $q_{u}$ $\left.=q_{v}=\pi\right)$. After solving the quadratic equation, we obtain for the MI gain,

$$
G \equiv \operatorname{Im}[K]= \pm \frac{1}{\sqrt{2}} \operatorname{Im}\left[\left(-a_{1} \pm \sqrt{a_{1}^{2}-4 a_{2}}\right)^{1 / 2}\right] .
$$

For $B=0$, expression (4) is close to the MI gain for the two-component coupled discrete fields of Refs. [32,33]. The effect of the FWM term is to decrease the necessary power to observe the modulational instability. The energy exchange between orthogonal polarizations improve the instability of plane waves allowing the formation of localized structures like discrete vector solitons.

To identify the regions of instability, in Fig. 2 we display the results of our analysis in the form of a contour plot in $\left(u_{0}, v_{0}\right)$ space where the more clear regions correspond to larger values of the MI gain, whereas darker regions correspond to lower or zero MI gain. In Fig. 2 the coupling parameter is weak, $C=0.1$, and we can see that discrete solitons are expected for $u_{0}=v_{0} \geq 0.4$.
The results for MI in the vectorial case that follow from Eq. (4) can be shown to reduce to the results for the scalar case studied earlier [31]. Indeed, taking one of the amplitudes equal to zero, or the nonlinear couplings $A=B=0$, or by taking $B=0$ and imposing that both modes possess equal amplitude, we obtain $a_{2}=0$ and $G= \pm \sqrt{a_{1}}$, and the expression reduces to the scalar MI gain from Ref. [31],

$$
G= \pm\left\{4 C \sigma_{u} \sin ^{2}\left(\frac{Q}{2}\right)\left[2 u_{0}^{2}-4 C \sigma_{u} \sin ^{2}\left(\frac{Q}{2}\right)\right]\right\}^{1 / 2} .
$$

Having identified the regions of modulational instability, we proceed now with the analysis of the localized modes, the energy transfer between the polarizations, and the calculation of the PN barrier that controls the transversal dynamics of the discrete solitons. To keep the description simple and obtain closed-form analytical expressions, we restrict our study by the case of strongly localized modes (SLM). This restriction to SLMs is, however, of great technological importance when trying to control the power exchange and steering of these modes for all-optical computing or communications purposes. Therefore, our focus is primarily on narrow optical solitons.

\section{STRONGLY LOCALIZED MODES}

First, we find the profiles of strongly localized modes of the simplest odd symmetry. We use the standard ansatz,

$$
\begin{aligned}
& u_{n}(z) \approx U\left\{\ldots, 0, \varepsilon e^{i k_{u}}, 1, \varepsilon e^{-i k_{u}}, 0, \ldots\right\} e^{i \lambda_{u^{z}}}, \\
& v_{n}(z) \approx V\left\{\ldots, 0, \delta e^{i k_{v}}, 1, \delta e^{-i k_{v}}, 0, \ldots\right\} e^{i \lambda_{v} z},
\end{aligned}
$$

where $U$ and $V$ are the amplitudes, $k_{u}$ and $k_{v}$ are the transverse components of the wave vectors (which describe initial kicks applied to the components), the parameters $\varepsilon$ and $\delta$ characterize the mode decay for the TE and TM components, and they are assumed to be small. After substituting this ansatz into Eq. (1) and keeping only the first-order terms in $\epsilon$ and $\delta$, we obtain

$$
\varepsilon \approx \frac{C \cos \left(k_{u}\right)}{U^{2}+(A+B) V^{2}}, \quad \delta \approx \frac{C \cos \left(k_{v}\right)}{V^{2}+(A+B) U^{2}} .
$$

Similarly, we find the profiles of strongly localized even modes, using the other ansatz,

$$
\begin{aligned}
& \bar{u}_{n} \approx \bar{U}\left\{\ldots, 0, \bar{\varepsilon} e^{i k_{u}}, 1, e^{-i k_{u}}, \bar{\varepsilon} e^{-2 i k_{u}}, 0, \ldots,\right\} e^{i \lambda_{u^{z}}^{\bar{z}}}, \\
& \bar{v}_{n} \approx \bar{V}\left\{\ldots, 0, \bar{\delta} e^{i k_{v}}, 1, e^{-i k_{v}}, \bar{\delta} e^{-2 i k_{v}}, 0, \ldots,\right\} e^{i \lambda_{v}^{-} \bar{z}},
\end{aligned}
$$

where $\bar{U}$ and $\bar{V}$ are the mode amplitudes, $k_{u}$ and $k_{v}$ are the transverse components of the wave vectors, $\bar{\varepsilon}$ and $\bar{\delta}$ are small parameters. To first order we obtain

$$
\bar{\varepsilon} \approx \frac{C \cos \left(k_{u}\right)}{C \cos \left(k_{u}\right)+\bar{U}^{2}+(A+B) \bar{V}^{2}},
$$




$$
\bar{\delta} \approx \frac{C \cos \left(k_{v}\right)}{C \cos \left(k_{v}\right)+\bar{V}^{2}+(A+B) \bar{U}^{2}} .
$$

From Eqs. (7) and (8), it follows that our assumptions that all parameters $\epsilon, \delta, \widetilde{\epsilon}, \widetilde{\delta}$ are small can always be satisfied for small values of the coupling parameter and high enough mode amplitudes.

We varied both, the mode amplitudes $U=V$, and the coupling parameter $C$, and confirmed numerically that the threesite analytical solutions approximate well the modes with large enough amplitudes or weak enough waveguide coupling. These modes display stability properties in agreement with Fig. 2. A complete analysis of the families of localized modes and their linear stability is still an open problem, and it is beyond the main scope of this paper.

\section{POWER TRANSFER}

\section{A. General comments}

The analysis presented above corresponds to the symmetric mode profiles and stable mode propagation. However, similar to the continuous cases of two-mode polarization dynamics [21-25], the TE and TM polarization modes exchange their powers while traveling along a waveguide array. Moreover, this effect can be reversed if we change the initial phase difference between the polarizations at the array input.

In order to get a deeper insight into the polarization instability dynamics and to study the mechanism of the power exchange, we study the partial powers in more detail. From conservation of the total power $P$, it follows that

$$
\frac{\partial P}{\partial z}=0 \Rightarrow \frac{\partial P_{u}}{\partial z}=-\frac{\partial P_{v}}{\partial z},
$$

i.e., as one of the polarizations gains extra power the other one loses it in the very same amount. Using the dynamical Eqs. (1), it is easy to show that

$$
\begin{aligned}
\frac{\partial P_{u}}{\partial z}=-\frac{\partial P_{v}}{\partial z} & =\sum_{n}\left(u_{n}^{*} \frac{\partial u_{n}}{\partial z}+u_{n} \frac{\partial u_{n}^{*}}{\partial z}\right) \\
& =2 B \sum_{n} \operatorname{Im}\left[u_{n}(z)^{2} v_{n}^{*}(z)^{2}\right],
\end{aligned}
$$

which highlights the role played by the FWM coupling terms in the power exchange. In the absence of FWM effects, i.e., for $B=0$, there are two conservation laws, two conserved powers, while in the presence of $B$ these restrictions are relaxed and only the total power is conserved, opening the route for the exchange of powers between the components. Also, the change of the relative phase between the polarization modes along the propagation direction $z$ is important for the power exchange, if the relative phase is zero (e.g., both the fields can be treated as real), the power exchange is absent. Thus, the initial phase between the modes is a very important quantity that determines the polarization mode dynamics. In order to pursue this idea analytically and examine the power transfer mechanism in detail, we employ the approximation of strongly localized modes used before, in Sec. IV. This approximation is very useful because, aside from simplifying the mathematical problem considerably, it still describes correctly the dynamics of different kinds of very localized input states which can be realized in experiment.

\section{B. Analytical results}

From Eq. (10), we notice that in the limit of strongly localized modes, only the contribution of the central waveguide field is important while other terms are proportional to the small parameter $\varepsilon^{2} \delta^{2}$. Thus, in this approximation, $d P_{u} / d z \approx 2 B \operatorname{Im}\left[\left(u_{0} v_{0}^{*}\right)^{2}\right]$. Using SLMs, we pose $u_{n}(z)$ $=U\left\{\ldots, 0, \varepsilon e^{i k_{u}}, 1, \varepsilon e^{-i k_{u}}, 0, \ldots\right\} e^{i \lambda_{u} z+i \phi_{u}}$ and $v_{n}(z)=V\{\ldots, 0$, $\left.\delta e^{i k_{v}}, 1, \delta e^{-i k_{v}}, 0, \ldots\right\} e^{i \lambda_{v} z+i \phi_{v}}$, and where $\phi_{u}$ and $\phi_{v}$ are the initial phases of the modes.

We obtain,

$$
\begin{aligned}
\left(u_{0} v_{0}^{*}\right)^{2}= & U^{2} V^{2}\left[1+2 \epsilon \delta \cos \left(k_{u}-k_{v}\right)\right]^{2} \\
& \times \exp \left[2 i\left(\lambda_{u}-\lambda_{v}\right) z+2 i\left(\phi_{u}-\phi_{v}\right)\right] .
\end{aligned}
$$

This implies,

$$
\frac{d P_{u}}{d z} \approx 2 B U^{2} V^{2}[1+2 \epsilon \delta \cos (\Delta k)]^{2} \sin [2 \Delta \lambda z+2 \Delta \phi],
$$

where $\Delta \lambda \equiv \lambda_{u}-\lambda_{v}, \Delta k \equiv k_{u}-k_{v}$, and $\Delta \phi \equiv \phi_{u}-\phi_{v}$. After integrating this expression, we find the approximate power variation in $z$,

$$
\begin{aligned}
P_{u}(z) \approx & U^{2}+\frac{2 B U^{2} V^{2}(1+2 \epsilon \delta \cos (\Delta k))^{2}}{2 \Delta \lambda}[\cos (2 \Delta \phi) \\
& -\cos (2 \Delta \lambda z+2 \Delta \phi)] .
\end{aligned}
$$

On the other hand, to first order in $\epsilon, \delta$ and assuming small transversal momenta $k_{u}, k_{v}$, we find $\lambda_{u} \approx 1+(C / \epsilon)=1+U^{2}$ $+(A+B) V^{2}$, and $\lambda_{v} \approx-1+(C / \delta)=-1+V^{2}(A+B) U^{2}$. This implies, $\Delta \lambda \approx 2-(A+B-1)\left(U^{2}-V^{2}\right)$. Thus, the power variations in $z$ are approximately given by

$$
\begin{aligned}
P_{u}(z) \approx & U^{2}+\frac{2 B U^{2} V^{2}}{4-2(A+B-1)\left(U^{2}-V^{2}\right)}[\cos (2 \Delta \phi) \\
& -\cos (2 \Delta \lambda z+2 \Delta \phi)]+O\left(\epsilon^{2}, \delta \varepsilon\right), \\
P_{v}(z) \approx & V^{2}-\frac{2 B U^{2} V^{2}}{4-2(A+B-1)\left(U^{2}-V^{2}\right)}[\cos (2 \Delta \phi) \\
& -\cos (2 \Delta \lambda z+2 \Delta \phi)]+O\left(\delta^{2}, \delta \varepsilon\right) .
\end{aligned}
$$

From these, we find the spatial average output powers for both modes,

$$
\begin{aligned}
\left\langle P_{u}\right\rangle \approx & U^{2}+\frac{2 B U^{2} V^{2}}{4-2(A+B-1)\left(U^{2}-V^{2}\right)} \cos (2 \Delta \phi) \\
& +O\left(\varepsilon^{2}, \delta \varepsilon\right)
\end{aligned}
$$

$\left\langle P_{v}\right\rangle \approx V^{2}-\frac{2 B U^{2} V^{2}}{4-2(A+B-1)\left(U^{2}-V^{2}\right)} \cos (2 \Delta \phi)+O\left(\delta^{2}, \delta \varepsilon\right)$.

Equations (15) describe, in a very simple way, the dependence of the power transfer on the relative phase between the 

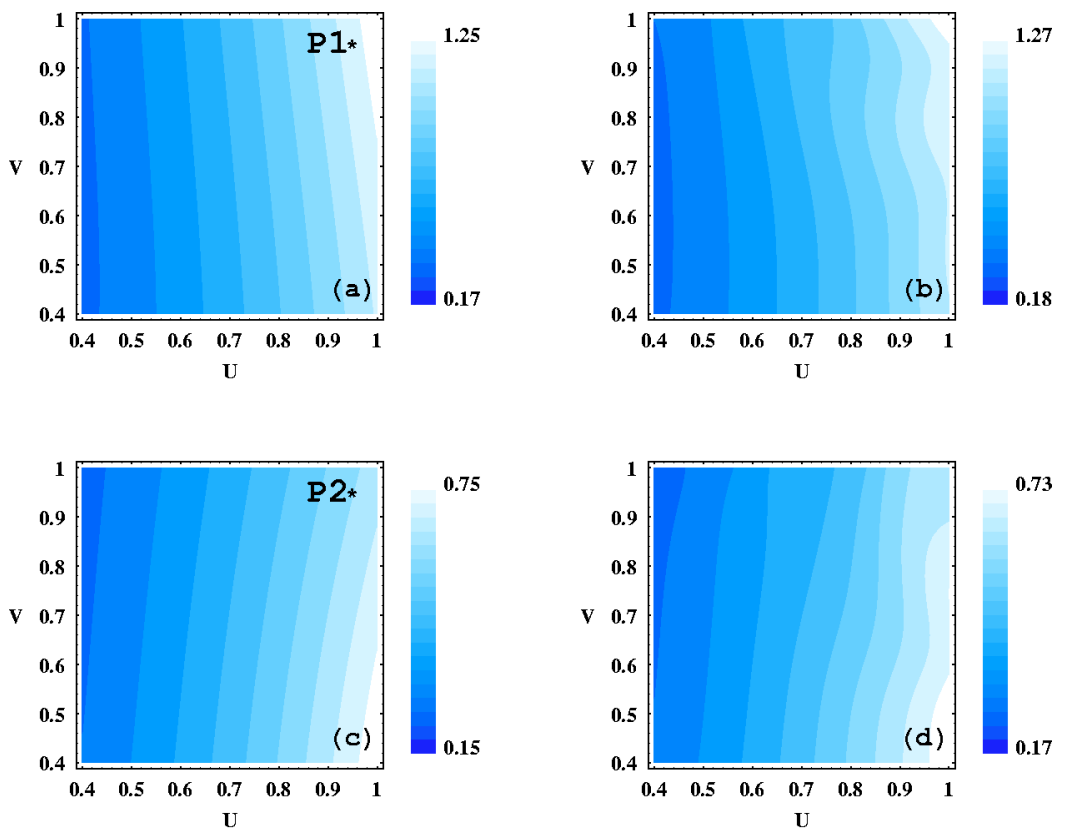

FIG. 3. (Color online) Comparison of the average TE output power as a function of the initial amplitudes $U$ and $V$, for $C=0.1$, and $z_{\max }=100$. Left, analytical predictions; right, numerical results. (a,b) $\Delta \phi=0,(\mathrm{c}, \mathrm{d}) \Delta \phi=\pi / 2$, (e,f) $\Delta \phi=\pi / 4$.
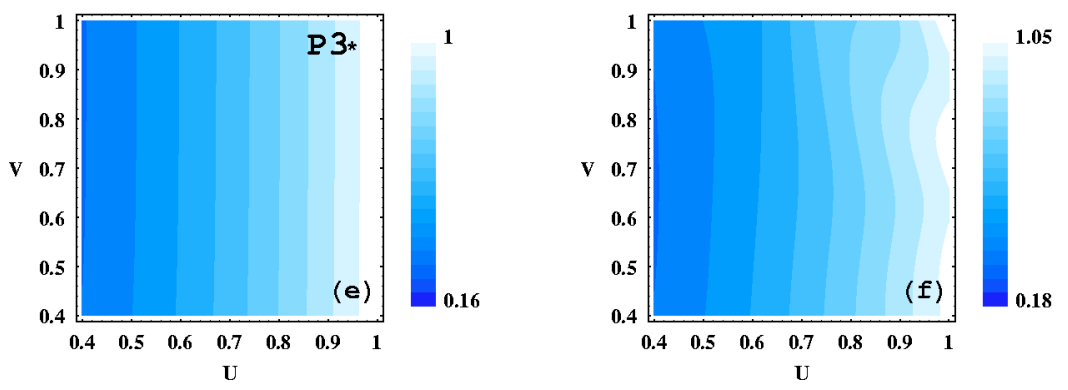

polarization modes. For the linear polarization (i.e., $\Delta \phi$ $=0, \pi)$, the TE mode gains extra power from the TM mode. On the other hand, if the initial polarization is elliptic $(\Delta \phi$ $=\pi / 2$ ), the power exchange process becomes reversed, and Eq. (15) shows that the TM mode gains some power from the TE mode.

\section{Numerical results}

In Fig. 3, we show the contour plots of the average TE output power for weak coupling $(C=0.1)$ as a function of the amplitudes of both modes. We present also a comparison between our theoretical predictions (left column) based on Eqs. (15), and direct numerical simulations (right column) based on integration of Eqs. (1). In Figs. 3(a) and 3(b), i.e., for the case of linear polarization, the tendency is that for bigger amplitudes of the modes, bigger TE output power is achieved. In this case, the gain for the TE mode from the TM mode is about $\sim 25 \%$. In Figs. 3(c) and 3(d), i.e., for the case of elliptic polarization, the TE output power is always lower than the TE input power, and the TM mode gains up to $\sim 25 \%$ of the TE mode power. In Figs. 3(e) and 3(f), there is no power gain, and this corresponds to the dynamics without exchange of power, on average. The three cases presented in Fig. 3 show a very good agreement between direct numerical results and our theoretical approximation, for the power transfer and the minimum and maximum TE power expected at the output. The average TM mode output power can be calculated as $\left\langle P_{v}\right\rangle \approx U^{2}+V^{2}-\left\langle P_{u}\right\rangle$.

In Figs. 3(a), 3(c), and 3(e), we marked three points labeled as P1, P2, and P3. For all of them, $U=V=0.95$. In Fig. 4 , we show the power evolution at these points in the $z$ direction. We display the dynamics for two $z$ intervals, from 0 to 5 (left-hand side), and from 95 to 100 (right-hand side). Our main idea is to compare the initial and final dynamics and observe if our approximation is good at the beginning and the end of the waveguide array. In this figure, we exhibit numerical (bold), theoretical (gray), and average theoretical (horizontal line gray) results for the power dynamics of the TE (solid) and TM (dashed) modes. Figure 4 shows a very good agreement between the theoretical and numerical results. In Fig. 4(a) (point P1), the TE mode acquires power from the TM mode at the output, for the case of linear initial polarization. Also, the average power prediction from Eqs. (15) seems to work very well. Figure 4(b) (point P2) shows the opposite power dynamics. For an elliptic initial polarization, the TM mode gains power from the TE mode. Also in this case, the average power prediction works very well, too. In Fig. 4(c) (point P3), the average power estimation is still in good agreement with the numerical results, but it fails to describe the details of the power dynamics.

The physics of the power transfer mechanism observed in the interaction between the TE and TM modes is explained 

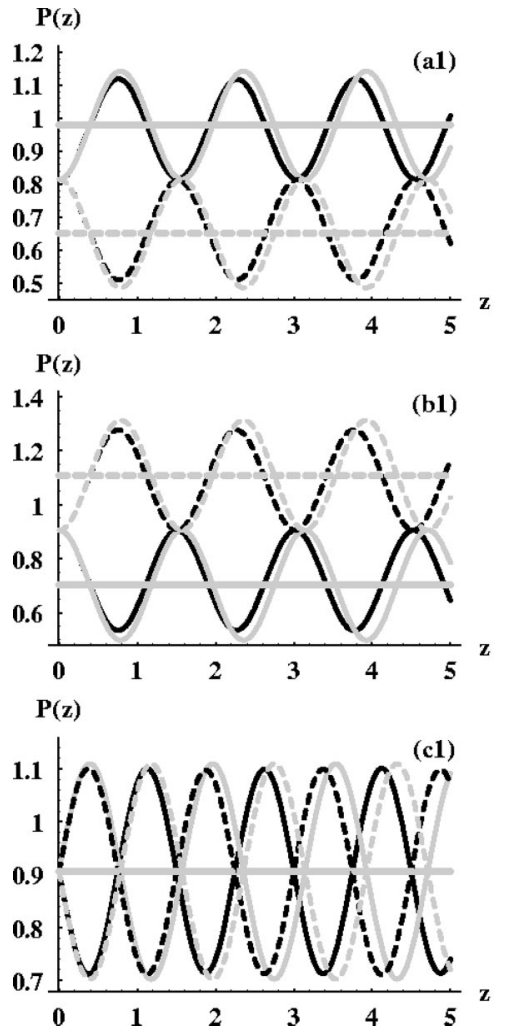
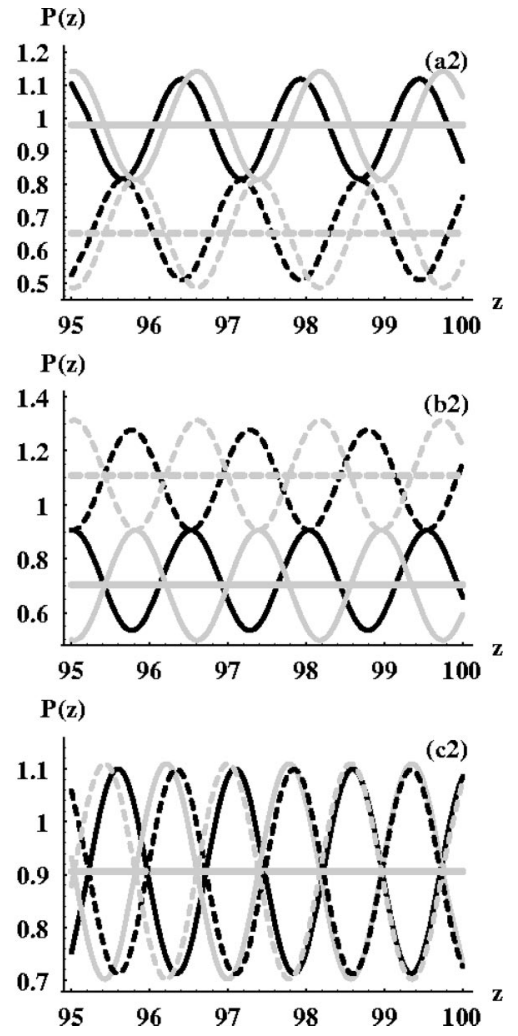

FIG. 4. Numerical (bold), analytical (gray), and average analytical (horizontal gray line) results for the power evolution of the TE (solid) and TM (dashed) modes, at $C=0.1$. Dynamics is shown in two different ranges of $z$. Left, $z=0-5$. Right, $z=95-100$. Cases (a), (b), and (c) represents the points P1, P2, and P3 in Figs. 3(a), 3(c), and $3(\mathrm{e})$, respectively. by the specific properties of birefringence of the cubic nonlinear response, and it is similar to the case of a homogeneous medium. If we consider only the linear terms of birefringence from Eqs. (1) for $n=0$, it is easy to show that the power variation for both modes has the form

$$
\frac{\partial P_{u}}{\partial z}=-\frac{\partial P_{v}}{\partial z} \approx \pm 2 B U^{2} V^{2} \sin (4 z),
$$

where the + and - signs represent the lineal and elliptic initial polarization cases, respectively. Now, let us exchange the birefringence. This implies $\mathrm{TE} \leftrightarrow \mathrm{TM} \Rightarrow$ fast wave $\rightarrow$ slow wave $\Rightarrow \sin (4 z) \rightarrow-\sin (4 z)$. As a result,

$$
\frac{\partial P_{u}}{\partial z}=-\frac{\partial P_{v}}{\partial z} \approx \mp 2 B U^{2} V^{2} \sin (4 z) .
$$

This demonstrates that birefringence plays an important role in the dynamics of the modes. Choosing an elliptical initial polarization, is the same as changing the sign of the birefringence, and therefore exchanging the slow and fast waves.

\section{PEIERLS-NABARRO POTENTIAL}

To understand the transversal dynamics of the discrete vector solitons, we calculate the Peierls-Nabarro barrier. Using the power and Hamiltonian from Eqs. (2) and (3), and assuming strong localization of the odd and even nonlinear modes described by Eq. (6) and Eq. (8), we can estimate the mode power and the Hamiltonian,

$$
\begin{gathered}
P_{\text {odd }} \approx U^{2}+V^{2}, \quad P_{\text {even }} \approx 2\left(\bar{U}^{2}+\bar{V}^{2}\right), \\
H_{\text {odd }} \approx-\left[U^{2}-V^{2}+\frac{1}{2}\left(U^{4}+V^{4}\right)+(A+B) U^{2} V^{2}\right],
\end{gathered}
$$

$$
\begin{aligned}
H_{\mathrm{even}} \approx & -2\left[\bar{U}^{2}-\bar{V}^{2}+\frac{1}{2}\left(\bar{U}^{4}+\bar{V}^{4}\right)+(A+B) \bar{U}^{2} \bar{V}^{2}\right. \\
& \left.+C\left(\bar{U}^{2}+\bar{V}^{2}\right) \cos k\right]
\end{aligned}
$$

where we take $k_{u}=k_{v}=k$. Next, and for the sake of simplicity, we take $U=V$ and $\bar{U}=\bar{V}$. Following a standard procedure [17-19], we consider the odd and even modes as two different states of the same nonlinear mode shifted by a half of the array period. This implies that the power should be the same for both modes, i.e., $P_{\text {odd }}=P_{\text {even }}$. With these assumptions, we evaluate the value of the Hamiltonian for these two modes, in terms of the mode amplitude $U$,

$$
\begin{gathered}
H_{\mathrm{odd}} \approx-(1+A+B) U^{4}, \\
H_{\mathrm{even}} \approx-\frac{1}{2}(1+A+B) U^{4}-2 C U^{2} \cos k .
\end{gathered}
$$

Finally, an estimate for the Peierls-Nabarro barrier is obtained by subtracting the values of both Hamiltonians, $\Delta H$ $\equiv H_{\text {odd }}-H_{\text {even }}$,

$$
\Delta H \approx-\frac{1}{2}(1+A+B) U^{4}+2 C U^{2} \cos k .
$$

This expression is similar to the scalar case discussed before [17], but it includes the effect of renormalization due to the cross-phase modulation coupling.

Result (19) means that at high powers and for small initial transversal momentum (or kick), the discrete vector soliton is not able to propagate across the waveguide array, because it needs to overtake the effective energy barrier which is very deep; the discrete soliton gets trapped at the input waveguide. At low powers and a finite initial kick, the effective trapping energy is low and the discrete soliton can travel 

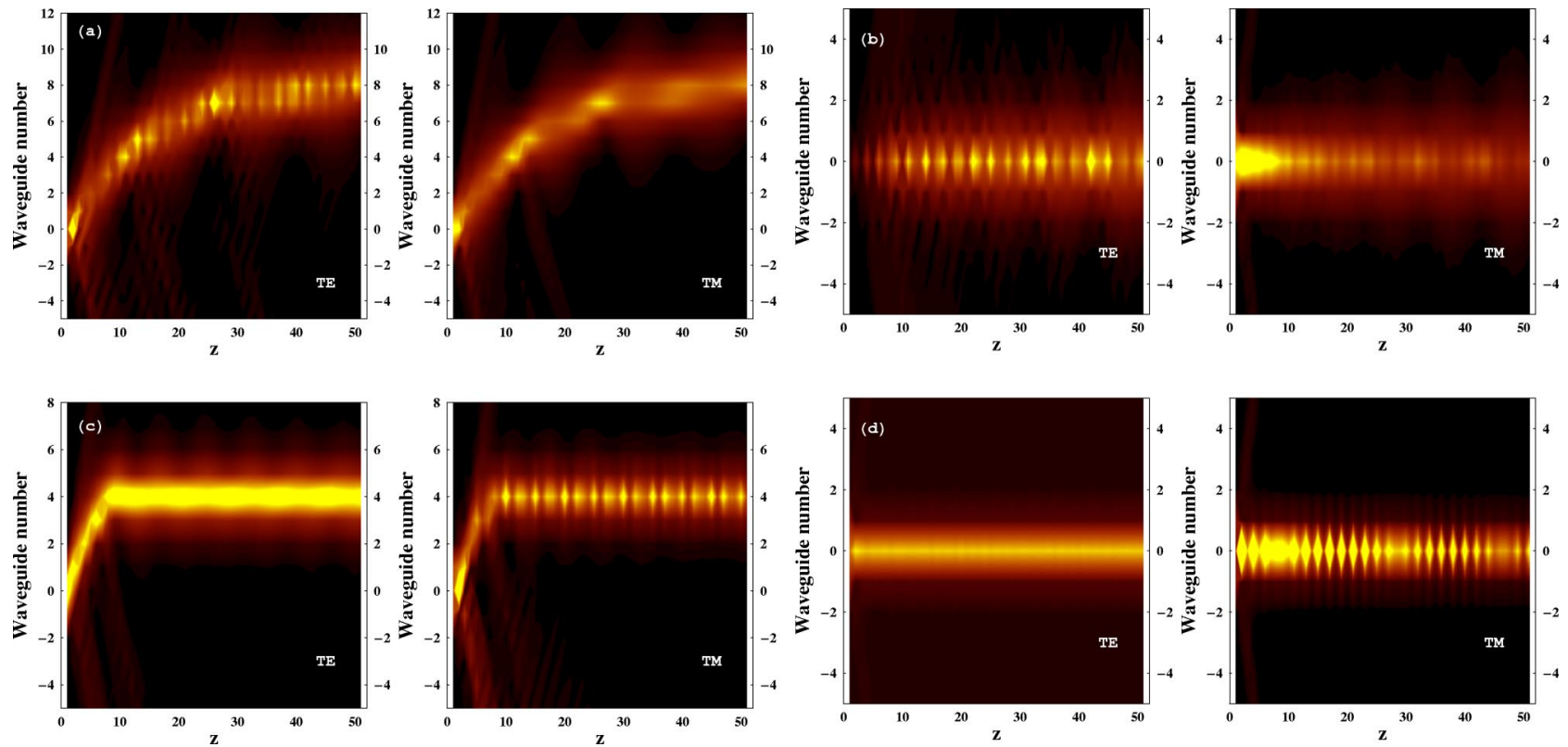

FIG. 5. (Color online) Examples of all-optical multiport switching based on discrete vector solitons. The coupling parameter is $C$ $=0.92$. Lineal polarization, (a) $k_{u}=0, k_{v}=-0.5, u_{0}=0.1$, and $v_{0}=1.248$, and (b) $k_{u}=k_{v}=0, u_{0}=0.1$, and $v_{0}=1.5$. Elliptic polarization, (c) $k_{u}$ $=-0.8, k_{v}=0, u_{0}=1.38$, and $v_{0}=0.1$, and (d) $k_{u}=k_{v}=0, u_{0}=1.5$, and $v_{0}=0.1$. Note that contour plots have different power scales for the TE and TM modes in each case.

across the waveguide array. At intermediate powers, the discrete soliton travels across the array for some distance from the input waveguide, and then gets trapped in some waveguide because of the nonlinearity. This kind of dynamics is a feature of nonlinear discrete systems, and it was recently suggested for multiport all-optical switching [16,17].

\section{MULTIPORT DISCRETIZED ALL-OPTICAL SWITCHING}

The results obtained so far suggest that there is a polarization instability where the flow of power could be directed by an appropriate choice of the initial relative phase between the TE and TM modes. Also, and due to the interplay of discreteness and nonlinearity, the lateral propagation of the optical soliton can be controlled by means of a judicious kick or amplitude tuning $[16,17]$. In this section we examine the combined switching or amplification of a narrow input beam, by solving Eq. (1) numerically. The beam profile is chosen in the form of a highly truncated sechlike profile $[16,17]$,

$$
\begin{aligned}
& u_{n}(0)=u_{0} \operatorname{sech}\left[u_{0}\left(n-n_{c u}\right) / \sqrt{2}\right] e^{-i k_{u}\left(n-n_{c u}\right)} e^{i \phi_{u}}, \\
& v_{n}(0)=v_{0} \operatorname{sech}\left[v_{0}\left(n-n_{c v}\right) / \sqrt{2}\right] e^{-i k_{v}\left(n-n_{c v}\right)} e^{i \phi_{v},}
\end{aligned}
$$

for $n-n_{c u}=n-n_{c v}=0, \pm 1$, and $u_{n}(0)=v_{n}(0)=0$, otherwise. Parameters $u_{0}$ and $v_{0}$ are the initial amplitudes, $k_{u}$ and $k_{v}$ are the initial kicks (the input angles), $n_{c u}$ and $n_{c v}$ are the initial soliton coordinates, and $\phi_{u}$ and $\phi_{v}$ are the initial mode phases for the TE and TM polarization components, respectively. From previous works $[16,17]$, we know that this ansatz works well for the case of scalar modes when the model is reduced to the discrete nonlinear Schrödinger (DNLS) equation. We point out that the choice of the initial input is not a crucial issue; the discrete soliton is a self-adjusting mode, and the energy excess is liberated in the form of radiation modes [23]. We have made numerical simulations with other profiles, such as strongly localized modes and discretized Gaussian modes, and the dynamic behavior is similar, although the sechlike input seems to work better.

The idea is to control a small TE (or TM) signal beam by means of a TM (or TE) kicked pump. In numerical simulations, we take $z_{\max }=50$ and an array of 110 waveguides. The value of the coupling parameter, $C=0.92$, is taken from recent experiments [29]. We use a small unkicked signal with an initial power of 0.03 [which corresponds to the dimension power $\sim 1.68$ (Watts)]. In the absence of a pump mode, the signal diffracts in the array and no discrete vector soliton is formed. In the presence of a strong pump, the power transfer is observed with the gain calculated as $G \equiv\left[\left\langle P_{u, v}\left(z_{\max }\right)\right\rangle\right.$ $\left.-P_{u, v}(0)\right] / P_{u, v}(0)$. The coupled dynamics of two polarization components leads to the formation of a discrete vector soliton that can be switched by varying the input kick.

Figure 5 shows results of multiport switching through the coupling of the TE and TM modes. Figure 5(a) shows an example of the eight-site switching. In this case, the gain power for the TE mode is of $\sim 90 \%$. In Fig. 5(b) there is no switching, and the gain for the TE mode is very high, $\sim 6000 \%$. A four-site switching is shown in Fig. 5(c) where the initial polarization is elliptical. The gain for the TM mode is $\sim 45 \%$. Finally, Fig. 5(d) shows no switching of both modes, with the gain of $\sim 30 \%$.

\section{CONCLUSIONS}

We have studied the properties of discrete vector solitons in arrays of weakly coupled birefringent optical waveguides 
with cubic nonlinear response. We have analyzed the effects of vectorial interaction on modulational instability, and obtained the profiles of strongly localized nonlinear modes. We have studied the polarization mode instability in the case of strongly localized solitons and demonstrated that the instability and power exchange between the TE and TM polarization components can be controlled by choosing the initial phase. We have calculated the effective Peierls-Nabarro potential for the discrete vector solitons and demonstrated how to employ the concepts of polarization instability and discreteness-induced beam trapping for achieving simultaneous switching and amplification of a weak signal by a strong pump of the other polarization.

\section{ACKNOWLEDGMENTS}

One of the authors (R.V.) acknowledges support from Conicyt. Two of the authors (M.M. and Y.K.) acknowledge support from Fondecyt Grants Nos. 1020139 and 7020139.
[1] H. S. Eisenberg, Y. Silberberg, R. Morandotti, A. R. Boyd, and J. S. Aitchison, Phys. Rev. Lett. 81, 3383 (1998).

[2] J. W. Fleisher, T. Carmon, M. Segev, N. K. Efremidis, and D. N. Christodoulides, Phys. Rev. Lett. 90, 023902 (2003); D. Neshev, E. A. Ostrovskaya, Yu. S. Kivshar, and W. Krolikowski, Opt. Lett. 28, 710 (2003).

[3] A. Fratalocchi, G. Assanto, K. A. Brzdakiewicz, and M. A. Karpierz, Opt. Lett. 29, 1530 (2004).

[4] F. Lederer and Y. Silberberg, Opt. Photonics News 13, 49 (2002); A. A. Sukhorukov, Yu. S. Kivshar, H. S. Eisenberg, and Y. Silberberg, IEEE J. Quantum Electron. 39, 31 (2003); D. N. Christodoulides, F. Lederer, and Y. Silberberg, Nature (London) 424, 817 (2003); F. Lederer and S. Aitchison, in Optical Solitons: Theoretical Challenges and Industrial Perspectives, edited by V. E. Zakharov and S. Wabnitz (Springer, Berlin, 1999), p. 349; P. G. Kevrekidis, K. Ø. Rasmussen, and A. R. Bishop, Int. J. Mod. Phys. B 15, 2833 (2001); F. Lederer, S. Darmanyan, and A. Kobyakov, in Spatial Solitons, edited by S. Trillo and W. Torruellas (Springer-Verlag, Berlin, 2001), p. 269; J. C. Eilbeck and M. Johansson, in Proceedings of the 3rd Conference Localization and Energy Transfer in Nonlinear Systems, June 17-21 2002, San Lorenzo de El Escorial Madrid, edited by L. Vázquez et al. (World Scientific, New Jersey, 2003), p. 44.

[5] D. N. Christodoulides and R. I. Joseph, Opt. Lett. 13, 794 (1988).

[6] Yu. S. Kivshar and G. P. Agrawal, Optical Solitons: From Fibers to Photonics Crystals (Academic, San Diego, 2003).

[7] D. K. Campbell, S. Flach, and Yu. S. Kivshar, Phys. Today 57 (1), 43 (2004).

[8] A. Trombettoni and A. Smerzi, Phys. Rev. Lett. 86, 2353 (2001); F. Kh. Abdullaev, E. N. Tsoy, B. A. Malomed, and R. A. Kraenkel, Phys. Rev. A 68, 053606 (2003).

[9] See, e.g., G. P. Tsironis, Chaos 13, 657 (2003), and references therein.

[10] B. I. Swanson, J. A. Brozik, S. P. Love, G. F. Strouse, A. P. Shreve, A. R. Bishop, W. Z. Wang, and M. I. Salkola, Phys. Rev. Lett. 82, 3288 (1999).

[11] U. T. Schwarz, L. Q. English, and A. J. Sievers, Phys. Rev. Lett. 83, 223 (1999).

[12] P. Binder, D. Abraimov, A. V. Ustinov, S. Flach, and Y. Zolotaryuk, Phys. Rev. Lett. 84, 745 (2000); E. Trias, J. J. Mazo, and T. P. Orlando, ibid. 84, 741 (2000).

[13] M. Sato, B. E. Hubbard, A. J. Sievers, and B. Ilic, Phys. Rev.
Lett. 90, 044102 (2003).

[14] W. Królikowski and Yu. S. Kivshar, J. Opt. Soc. Am. B 13, 876 (1996).

[15] T. Pertsch, U. Peschel, and F. Lederer, Opt. Lett. 28, 102 (2003).

[16] R. A. Vicencio, M. I. Molina, and Yu. S. Kivshar, Opt. Lett. 28, 1942 (2003).

[17] R. A. Vicencio, M. I. Molina, and Yu. S. Kivshar, Phys. Rev. E 70, 026602 (2004).

[18] Yu. S. Kivshar and D. K. Campbell, Phys. Rev. E 48, 3077 (1993).

[19] L. Hadzievski, A. Maluckov, M. Stepic, and D. Kip, Phys. Rev. Lett. 93, 033901 (2004).

[20] Y. Kartashov, A. Zelenina, L. Torner, and V. Vysloukh, Opt. Lett. 29, 766 (2004).

[21] K. J. Blow, N. J. Doran, and D. Wood, Opt. Lett. 12, 202 (1987).

[22] N. N. Akhmediev and J. M. Soto-Crespo, Phys. Rev. E 49, 5742 (1994).

[23] C. M. de Sterke and J. E. Sipe, Opt. Lett. 16, 202 (1991).

[24] E. A. Ostrovskaya, N. N. Akhmediev, G. I. Stegeman, J. U. Kang, and J. S. Aitchison, J. Opt. Soc. Am. B 14, 880 (1997).

[25] R. Malendevich, L. Friedrich, G. I. Stegeman, J. M. SotoCrespo, N. N. Akhmediev, and J. S. Aitchison, J. Opt. Soc. Am. B 19, 695 (2002).

[26] A. Schauer, I. V. Melnikov, and J. S. Aitchison, J. Opt. Soc. Am. B 21, 57 (2004).

[27] X. D. Cao and D. D. Meyerhofer, Opt. Lett. 19, 1711 (1994).

[28] S. Darmanyan, A. Kobyakov, E. Schmidt, and F. Lederer, Phys. Rev. E 57, 3520 (1998).

[29] J. Meier, J. Hudock, D. N. Christodoulides, G. I. Stegeman, Y. Silberberg, R. Morandotti, and J. S. Aitchison, Phys. Rev. Lett. 91, 143907 (2003).

[30] J. U. Kang, G. I. Stegeman, A. Villeneuve, and J. S. Aitchison, Pure Appl. Opt. 5, 583 (1996).

[31] Yu. S. Kivshar and M. Peyrard, Phys. Rev. A 46, 3198 (1992); S. Darmanyan, I. Relke, and F. Lederer, Phys. Rev. E 55, 7662 (1997).

[32] A. Kobyakov, S. Darmanyan, F. Lederer, and E. Schmidt, Opt. Quantum Electron. 30, 795 (1998).

[33] Z. Rapti, A. Trombettoni, P. G. Kevrekidis, D. J. Frantzeskakis, B. A. Malomed, and A. R. Boshop, Phys. Lett. A 330, 95 (2004). 